\title{
PD-L1/IDO Peptide Vaccine
}

National Cancer Institute

\section{Source}

National Cancer Institute. PD-L1/IDO Peptide Vaccine. NCI Thesaurus. Code C148154.

A peptide vaccine composed of 10103 , a peptide vaccine derived from the tumorassociated antigen (TAA) programmed cell death-1 lig and 1 (PD-L1), IO102, the 21-mer peptide vaccine derived from the immunomodulatory enzyme indoleamine 2,3dioxygenase (IDO), and the immunoadjuvant montanide ISA-51, with potential immunomodulating and antineoplastic activities. Vaccination with the PD-L1/IDO peptide vaccine may activate the immune system to induce an immune response against PD-L1 and IDO-expressing cells. This may increase and restore the proliferation and activation of various immune cells, including cytotoxic T-lymphocytes (CT Ls), and may eradicate PD-L1 and IDO-expressing tumor cells through a CT L-mediated immune response. PDL1 is overexpressed on many human cancer cell types. PD-L1 binding to its cognate receptor prog rammed cell death protein 1 (PD-1; PDCD1; CD279) on T-cells suppresses the immune system and results in increased immune evasion and decreased CTL activation. IDO, a cytosolic enzyme responsible for tryptophan catabolism and conversion of tryptophan into kynurenine, is overexpressed by a variety of tumor cell types and antigen presenting cells (APCS) and plays an important role in immunosuppression mainly through suppression of CT L activation; tryptophan depletion inhibits T-lymphocyte proliferation and activation, and suppresses the immune system. Montanide ISA-51, also known as incomplete Freund's adjuvant or IFA, is a stabilized water-in-oil emulsion adjuvant containing mineral oil with mannide oleate added as a surfactant that non-specifically stimulates cell-mediated immune responses to antigens. 